\title{
Influence of Generalized Coordinates on System Dynamics
}

\author{
Altay Zhakatayev $^{1,2}$, Yuriy Rogovchenko ${ }^{1}$, Matthias Pätzold ${ }^{2}$ \\ ${ }^{1}$ Dept. of Mathematics \\ University of Agder \\ 4604 Kristiansand, Norway \\ ${ }^{2}$ Department of ICT \\ University of Agder \\ 4879 Grimstad, Norway \\ altay.zhakatayev@uia.no,yuriy.rogovchenko@uia.no \\ matthias.paetzold@uia.no
}

\begin{abstract}
We investigate the effect of the choice of a set of generalized coordinates (GCs) on the simulation of the behavior of the dynamical system using the single-link spherical pendulum as an example. Specifically, we focus our attention on numerical errors and the simulation time necessary to simulate system dynamics. The Lagrangian method is applied to obtain the equations of motion. The generalized Euler angles are used as GCs. The GCs depend on the direction of the axes along which they are defined. Therefore, by parameterizing the directions of these two axes, different sets of GCs with the corresponding system of nonlinear differential equations are obtained. For a spherical pendulum, we demonstrate that the optimal sets of GCs leading to the minimum simulation time are orthogonal sets. However, contrary to our expectations, orthogonal sets do not result in the minimum simulation error. Additionally, the intrinsic generalized Euler angles lead to faster simulations than the extrinsic ones. Therefore, different choices of GCs are not equivalent from a numerical point of view and further research is needed to develop a strategy for selecting an optimal set of GCs.
\end{abstract}

Keywords: Generalized Coordinates, Davenport Angles, Lagrangian Dynamics, Optimal Generalized Coordinates, Multibody Systems Dynamics.

\section{INTRODUCTION}

Dynamic equations describing the evolution of systems can be derived using the Newton-Euler, Lagrangian, Hamiltonian method, or other formalisms [1]. The Newton-Euler formalism employs Cartesian coordinates for the position vector. In contrast, Lagrangian and Hamiltonian mechanics utilize generalized coordinates (GCs), which can be any set of variables suitable to fully describe the configuration of a system, e.g., Cartesian coordinates, relative and absolute angles, linear and angular momentum, and energy [2]. The number of variables is often chosen to be equal to the number of degrees of freedom (DOF) of the system, but it may also contain more variables than necessary. From a theoretical point of view, all possible sets of GCs are equally valid for describing system dynamics [3]. However, from a numerical or computational point of view, there can be differences. For simple systems with a few DOF, it is expected that there will be no discernible computational differences with regard to the choice of a set of GCs and efficiency is not an issue. However, for complex multi-body systems with a large number of DOF, the efficiency and computational time required to perform the analysis can vary greatly depending on the selected set of GCs.

A methodology of switching between different sets of GCs for multi-DOF planar mechanical systems was considered in [4]. The authors were primarily concerned with the development of a mathematical description for deriving control equations in a direct and transparent manner. Using Cartesian coordinates and the full row rank property of the constraint Jacobian matrix formed by independent constraints, Wehage and Haug [5] proposed a numerical criterion to obtain the minimum set of GCs. The method was extended for nonholonomic systems in [6]. Later work in this direction can be found in [7,8]. Efficient formulation of dynamic equations was the topic 
in [9-12]. Still, these works did not compare the effect of different sets of GCs on the solution of dynamic equations. In addition, there is a gap in the literature regarding the choice of optimal sets of GCs that lead to the numerical solution of differential equations with minimal error within the shortest simulation time. To the best of the authors' knowledge, there are no qualitative rules for selecting the optimal set of GCs [13]. As a result, there is a need in modern engineering for deeper investigations of the choice of GCs for describing the system dynamics.

In this work, our main motivation is to contribute to the investigation of the influence of a choice of GCs on the numerical solution of dynamic equations. The main objective of this paper is to understand the dependence of simulation error and time on the selected set of GCs. Another objective is to find the optimal set of GCs for a single-link spherical pendulum, which is considered as a test system. It is widely accepted that the problem-solving-experience is necessary to select the optimal set of GCs [13] and the search for a procedure which helps to identify the optimal set of GCs for a given system still continues.

The main novelty of this paper is the analysis of the dependence of the overall simulation error and computation time on the selected set of GCs based on the parameterized Davenport angles [14]. The main contributions of our work are to show that there are optimal sets of GCs for the spherical pendulum, and that choice of a GCs set has an impact on the simulations. Our principal results are the following. First, the choice of GCs strongly affects the simulation time and accuracy. Second, the optimal sets of GCs that minimize the simulation time do not coincide with those that minimize the gross simulation error. Third, the intrinsic Davenport angles appear to be more optimal for the spherical pendulum than extrinsic ones.

The paper is organized as follows. Sect. 2 gives a brief description of dynamic equations of motion. The kinematics of the spherical pendulum used as a test bed system is discussed in Sect. 3, where four types of sets of GCs are considered. Sect. 4 describes the simulations settings, and the simulations results are presented in Sect. 5. Finally, Sect. 6 presents the conclusions.

\section{DYNAMIC EQUATIONS OF MOTION}

In this paper, we develop dynamic equations of motion using the Lagrangian formalism. Let us define a set of GCs as $\mathbf{q} \in \mathbb{R}^{n}$, where $n$ is the number of DOF of a mechanical system under consideration, and $\mathbf{q}=\left[q_{1}, q_{2}, \ldots, q_{n}\right]^{T}$ is a column vector. Here, the transpose operator is denoted as $[\cdot]^{T}$. The corresponding set of generalized velocities (GVs) $\dot{\mathbf{q}} \in \mathbb{R}^{n}$ is defined as the time derivative of the GCs, i.e., $\dot{\mathbf{q}}=\left[\dot{q}_{1}, \dot{q}_{2}, \ldots, \dot{q}_{n}\right]^{T}$, where the overdot denotes the time differentiation operator. Equations of motion of a mechanical system can often be written in the form

$$
M(\mathbf{q}) \ddot{\mathbf{q}}+\mathbf{C}(\mathbf{q}, \dot{\mathbf{q}})+\mathbf{G}(\mathbf{q})=\mathbf{Q}
$$

where $M(\mathbf{q}) \in \mathbb{R}^{n \times n}$ is the symmetric inertia matrix, $\mathbf{C}(\mathbf{q}, \dot{\mathbf{q}}) \in \mathbb{R}^{n}$ is the vector of Coriolis and normal inertial forces, and the vector $\mathbf{G}(\mathbf{q}) \in \mathbb{R}^{n}$ describes the effect of gravity. The vector of generalized forces is $\mathbf{Q}=\left[Q_{1}, Q_{2}, \ldots, Q_{n}\right]^{T}$. The dynamic equation (1) comprises a set of $n$ second-order differential equations.

To solve these equations with numeric solvers or to tackle a control problem of a mechanical system, it is desirable to have a system of $2 n$ first-order differential equations instead. As a result, Eq. (1) is further modified to the so-called state-space form

$$
\dot{\mathbf{x}}=\mathbf{f}(\mathbf{x}, \mathbf{Q})
$$

where $\mathbf{x}=\left[\mathbf{q}^{T}, \dot{\mathbf{q}}^{T}\right]^{T} \in \mathbb{R}^{2 n}$ is the state vector, and $\mathbf{Q}$ serves as the control input vector. The nonlinear state function $\mathbf{f}$ can be written as

$$
\mathbf{f}(\mathbf{x}, \mathbf{Q})=\left[\begin{array}{c}
\dot{\mathbf{q}} \\
-M^{-1}(\mathbf{C}(\mathbf{q}, \dot{\mathbf{q}})+\mathbf{G}(\mathbf{q})-\mathbf{Q})
\end{array}\right] .
$$


Our task now is to select a mechanical system and different sets of GCs that describe its kinematics. Then, for the selected sets of GCs, we formulate and solve the dynamic equations in Eq. (2), where $\mathbf{f}$ is defined by (3).

\section{KINEMATICS OF A SPHERICAL PENDULUM}

As our test system, we consider a single-link spherical pendulum which is symmetric with respect to the rotation about its axis, as shown in Fig. 1a. We intentionally chose the spherical pendulum because its motion is three-dimensional, while the motion of a simple pendulum is only twodimensional. Although in reality the spherical pendulum still has a limited range of angular motion, we can assume that there are no restrictions on the range of its motion. Three angles are required to correctly orient a three-dimensional object in the three-dimensional space. However, due to rotational symmetry around its own symmetry axis, only two angles are used to fully specify the configuration (orientation) of the pendulum in our model. This pair of angles will be denoted as $q_{1}$ and $q_{2}$. The Euler angles were generalized to rotations with respect to non-orthogonal axes in [14], also called Davenport angles [15,16]. In this work, Davenport angles are used as GCs. The mass, inertia matrix about the center of mass, length, and the length up to the center of mass of the pendulum will be designated by $m, I, L$, and $L_{c}$, respectively.

In the following, the Denavit-Hartenberg (DH) convention will be utilized to derive the kinematics of the pendulum [17,18]. The fixed global axes $X, Y, Z$ (Fig. 1) are used later to visualize the simulation results. The local axes $x_{i}, y_{i}, z_{i}, i=0,1,2$, define a frame $i$. These frames are used to derive the kinematics with the DH convention. To simplify the visualization, the local $y_{i}$ axes are not shown in Fig. 1. The local axes $x_{0}, y_{0}, z_{0}$ are also fixed. Even if they might seem redundant due to the presence of $X, Y, Z$, their introduction facilitates the derivation. The counterclockwise rotation angles of $q_{1}$ and $q_{2}$ are determined by the right-hand rule. The origin of the local coordinate system 2 can be located either at the end of the link or at its center of mass. In the derivations, the latter is chosen, but in Fig. 1, the former is used for the presentation clarity. In what follows, we will consider four cases with different sets of GCs.

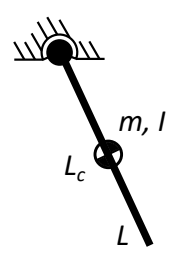

(a)

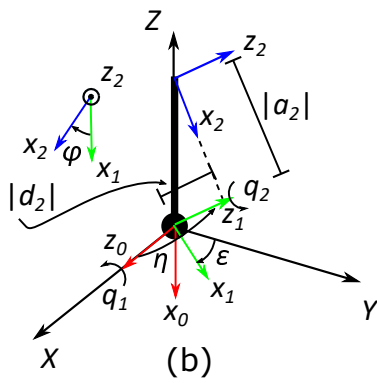

(b)

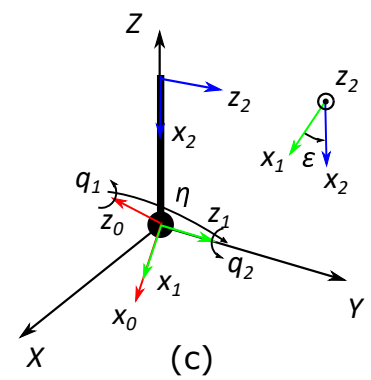

(c)

Figure 1: Schematic diagrams of the spherical pendulum (a), its kinematics with coordinate frames, angular parameters, and GCs for Cases I, III (b) and Cases II, IV (c).

\subsection{Case I}

In the first case, we assume that the axis of the first GC $q_{1}$ is aligned along the global axis $X$, while the axis of the second GC has an arbitrary initial direction with respect to the global axes (see Fig. 1b). The rotations are assumed to be intrinsic. Due to the rotation around the local axis, the direction of $q_{2}$ is affected by $q_{1}$. There are three angular parameters for this system: $\eta$ - the angle between $z_{0}$ and $z_{1}$ with respect to the axis $x_{1}, \varepsilon-$ the angle between $x_{1}$ and $Y$ with respect to the axis $X$, and $\varphi$ - the angle between $x_{1}$ and $x_{2}$ with respect to the axis $z_{2}$. We note that the unit vector $x_{1}$ is in the $Y Z$ plane. Additionally, there are two spatial parameters $d_{2}$ and $a_{2}$, which are used in 
the DH derivations. The geometry results in the following identities for the system parameters

$$
\begin{aligned}
& d_{2}=L_{c} s(\eta) c(\varepsilon), \\
& a_{2}=-L_{c} \sqrt{1-(s(\eta) c(\varepsilon))^{2}}, \\
& \varphi=\operatorname{atan} 2\left(-\frac{c(\eta) c(\varepsilon)}{\sqrt{1-(s(\eta) c(\varepsilon))^{2}}} ; \frac{s(\varepsilon)}{\sqrt{1-(s(\eta) c(\varepsilon))^{2}}}\right),
\end{aligned}
$$

where $s(\eta)$ and $c(\eta)$ are short notations for $\sin \eta$ and $\cos \eta$ functions, and similarly for the $\varepsilon$. Given $x$ and $y$, the output of the function atan2 $(y ; x)$ is the angle in the range $(-\pi, \pi]$. It can be observed from Eq. (4) that $\varphi, d_{2}$ and $a_{2}$ depend only on the other two parameters: $\eta$ and $\varepsilon$. The link and joint parameters for a spherical pendulum are summarized in Tab. 1 , where $\theta_{i}, d_{i}, a_{i}$, and $\alpha_{i}$ denote the corresponding DH parameters.

\subsection{Case II}

In the second case, it is assumed that the axis of the first GC $q_{1}$ is tilted with respect to the global frame, while the second GC $q_{2}$ has its initial direction aligned with respect to the global axis $Y$, as shown in Fig. 1c. Again, intrinsic rotations are assumed, so that the direction of the axis $q_{2}$ is influenced by the rotation $q_{1}$. There are two parameters in this system: $\eta$ - the angle between $z_{0}$ and $z_{1}$ with respect to the axis $x_{1}$, and $\varepsilon$ - the angle between $x_{1}$ and $x_{2}$ with respect to the axis $z_{2}$. We note that $x_{1}$ is in the $X Z$ plane. Similarly to Case I, the link and joint parameters for the spherical pendulum are listed in Tab. 1 .

The homogeneous transformation matrices [19] for the Cases I and II are obtained by substituting the DH parameters into the homogeneous transformation matrix ${ }^{i-1} A_{i} \in \mathbb{R}^{4 \times 4}$ from the local frame $i$ to the frame $i-1$ defined by

$$
{ }^{i-1} A_{i}=\left[\begin{array}{cccc}
c\left(\theta_{i}\right) & -c\left(\alpha_{i}\right) s\left(\theta_{i}\right) & s\left(\alpha_{i}\right) s\left(\theta_{i}\right) & a_{i} c\left(\theta_{i}\right) \\
s\left(\theta_{i}\right) & c\left(\alpha_{i}\right) c\left(\theta_{i}\right) & -s\left(\alpha_{i}\right) c\left(\theta_{i}\right) & a_{i} s\left(\theta_{i}\right) \\
0 & s\left(\alpha_{i}\right) & c\left(\alpha_{i}\right) & d_{i} \\
0 & 0 & 0 & 1
\end{array}\right]
$$

and including both translation and rotation. The position of the center of mass in the local bodyfixed frame 2 is ${ }^{2} \mathbf{r}_{2}=[0,0,0,1]^{T}$, because the origin coincides with the center of mass. With respect to the global frame 0 , the center of mass of the link is $\mathbf{r}_{2}=T_{2}{ }^{2} \mathbf{r}_{2}$, where $T_{2}={ }^{0} A_{1}{ }^{1} A_{2}$ is the combined homogeneous transformation matrix from the frame 2 to frame 0 . The potential energy of the link is $V=-m \mathbf{g}^{T} \mathbf{r}_{2}$, where $\mathbf{g}=\left[g_{x}, g_{y}, g_{z}, 0\right]^{T}$ and $g_{x}, g_{y}, g_{z}$ are the components of the gravitational constant $9.81 \mathrm{~m} / \mathrm{s}^{2}$ in the frame 0 . For instance, in Case I, $\mathbf{g}=9.81[1,0,0,0]^{T}$, while in Case II, $\mathbf{g}=9.81[c(\varepsilon), c(\eta) s(\varepsilon),-s(\eta) s(\varepsilon), 0]^{T}$.

The linear velocity of the center of mass in the global frame 0 can be obtained as $\mathbf{v}_{2}=\dot{\mathbf{r}}_{2}=\dot{T}_{2}{ }^{2} \mathbf{r}_{2}$. The translational kinetic energy is $K_{t}=1 / 2 m \mathbf{v}_{2}^{2}$. The angular velocity of the link around its center of mass in the body-fixed frame 2 is ${ }^{2} \omega_{2}=T_{2}^{T} \mathbf{q}_{1}+{ }^{1} A_{2}^{T} \mathbf{q}_{2}$, where $\mathbf{q}_{i}=\left[0,0, \dot{q}_{i}, 0\right]^{T}$ for $i=1,2$. Then, the rotational kinetic energy is $K_{r}={ }^{2} \omega_{2}^{T}{ }^{2} J_{2}{ }^{2} \omega_{2} / 2$, where ${ }^{2} J_{2} \in \mathbb{R}^{4 \times 4}$ is the inertia matrix in the body-fixed frame 2 . This matrix can be written as

$$
{ }^{2} J_{2}=\left[\begin{array}{cccc}
I_{x x} & I_{x y} & I_{x z} & 0 \\
I_{y x} & I_{y y} & I_{y z} & 0 \\
I_{z x} & I_{z y} & I_{z z} & 0 \\
0 & 0 & 0 & 0
\end{array}\right]=\left[\begin{array}{cc}
{ }^{2} I_{2} & \mathbf{0} \\
3 \times 3 & 3 \times 1 \\
\mathbf{0} & 0 \\
1 \times 3 &
\end{array}\right] .
$$

The total kinetic energy is $K=K_{t}+K_{r}$. Both $K$ and $V$ are the functions of GCs $q_{1}, q_{2}$, and the parameters $\eta, \varepsilon$. 


\begin{tabular}{|c|c|c|c|c|c|c|c|c|c|}
\hline \multicolumn{7}{|c|}{ Case I } & \multicolumn{7}{c|}{ Case II } \\
\hline Joint, $i$ & $\theta_{i}$ & $d_{i}$ & $a_{i}$ & $\alpha_{i}$ & Joint, $i$ & $\theta_{i}$ & $d_{i}$ & $a_{i}$ & $\alpha_{i}$ \\
\hline 1 & $\frac{\pi}{2}-\varepsilon+q_{1}$ & 0 & 0 & $-\eta$ & 1 & $q_{1}$ & 0 & 0 & $-\eta$ \\
\hline 2 & $\phi+q_{2}$ & $d_{2}$ & $a_{2}$ & 0 & 2 & $\varepsilon+q_{2}$ & 0 & $-L_{c}$ & 0 \\
\hline
\end{tabular}

Table 1: DH parameters and variables for a spherical pendulum.

\subsection{Case III}

Geometrically, this case is similar to Case I, but the rotations $q_{1}$ and $q_{2}$ are assumed to be extrinsic. In other words, the axes of the rotation of $q_{1}$ and $q_{2}$ are fixed with respect to the global frame X-Y-Z. Therefore, the rotation $q_{1}$ does not affect the axis of rotation of $q_{2}$. Since the DH convention is only applicable to intrinsic rotations, in this subsection we use simple rotation matrices $R \in \mathbb{R}^{3 \times 3}$ to describe the kinematics.

The rotation matrix for $q_{1}$ will be a simple rotation matrix relative to the $z$-axis. From Fig. $1 \mathrm{~b}$, it can be observed that in the global frame 0 the unit vector $z_{1}=[-s(\eta) c(\varepsilon), s(\eta) s(\varepsilon), c(\eta)]^{T}$. Thus, both $z_{1}$ and $q_{2}$ define the principal rotation axis and the angle by which the rotation matrix ${ }^{1} R_{2}$ can be found

$$
{ }^{1} R_{2}=\left[\begin{array}{ccc}
k_{1} s^{2}(\eta) c^{2}(\varepsilon)+c\left(q_{2}\right) & -k_{1} s^{2}(\eta) s(\varepsilon) c(\varepsilon)-c(\eta) s\left(q_{2}\right) & -s(\eta)\left(k_{1} c(\eta) c(\varepsilon)-s(\varepsilon) s\left(q_{2}\right)\right) \\
-k_{1} s^{2}(\eta) s(\varepsilon) c(\varepsilon)+c(\eta) s\left(q_{2}\right) & k_{1} s^{2}(\eta) s^{2}(\varepsilon)+c\left(q_{2}\right) & s(\eta)\left(k_{1} c(\eta) s(\varepsilon)+c(\varepsilon) s\left(q_{2}\right)\right) \\
-s(\eta)\left(k_{1} c(\eta) c(\varepsilon)+s(\varepsilon) s\left(q_{2}\right)\right) & s(\eta)\left(k_{1} c(\eta) s(\varepsilon)-c(\varepsilon) s\left(q_{2}\right)\right) & k_{1} c^{2}(\eta)+c\left(q_{2}\right)
\end{array}\right] .
$$

where $k_{1}=1-c\left(q_{2}\right)$.

\subsection{Case IV}

This case is similar to Case II, but this time with the extrinsic angles $q_{1}$ and $q_{2}$. Similarly to Case III, $q_{1}$ does not influence the rotation axis of $q_{2}$. The rotation matrix for $q_{1}$ is obtained from the unit vector $z_{0}=[-s(\eta) s(\varepsilon), c(\eta), s(\eta) c(\varepsilon)]^{T}$ (see Fig. 1) which specifies the principal axis. Thus, the rotation matrix is

$$
{ }^{0} R_{1}=\left[\begin{array}{ccc}
k_{2} s^{2}(\eta) s^{2}(\varepsilon)+c\left(q_{1}\right) & -s(\eta)\left(k_{2} c(\eta) s(\varepsilon)+c(\varepsilon) s\left(q_{1}\right)\right) & -k_{2} s^{2}(\eta) s(\varepsilon) c(\varepsilon)+c(\eta) s\left(q_{1}\right) \\
-s(\eta)\left(k_{2} c(\eta) s(\varepsilon)-c(\varepsilon) s\left(q_{1}\right)\right) & k_{2} c^{2}(\eta)+c\left(q_{1}\right) & s(\eta)\left(k_{2} c(\eta) c(\varepsilon)+s(\varepsilon) s\left(q_{1}\right)\right) \\
-k_{2} s^{2}(\eta) s(\varepsilon) c(\varepsilon)-c(\eta) s\left(q_{1}\right) & s(\eta)\left(k_{2} c(\eta) c(\varepsilon)-s(\varepsilon) s\left(q_{1}\right)\right) & k_{2} s^{2}(\eta) c^{2}(\varepsilon)+c\left(q_{1}\right)
\end{array}\right],
$$

where $k_{2}=1-c\left(q_{1}\right)$. The rotation matrix for $q_{2}$ is a simple rotation matrix about the $y$-axis.

For Cases III and IV the overall rotation matrix is found as $R_{2}={ }^{1} R_{2}{ }^{0} R_{1}$ (in reverse order), due to the rotation sequence with respect to the global axes (extrinsic rotations). The position of the center of mass in the initial configuration is ${ }^{2} \mathbf{r}_{2}=\left[-L_{c}, 0,0\right]^{T}$. The same radius vector after the rotations becomes $\mathbf{r}_{2}=R_{2}{ }^{2} \mathbf{r}_{2}$ (expressed in the frame 0). By redefining $\mathbf{g}=[9.81,0,0]^{T}$, the potential energy of the link equals $V=-m \mathbf{g}^{T} \mathbf{r}_{2}$. By using the linear velocity of the link center of mass $\mathbf{v}_{2}=\mathbf{r}_{2}=\dot{R}_{2}{ }^{2} \mathbf{r}_{2}$, the translational kinetic energy is found as $K_{t}=1 / 2 m \mathbf{v}_{2}^{2}$. The angular velocities in the global frame are $\omega_{2}=\left[-s(\eta) c(\varepsilon) \dot{q}_{2}, s(\eta) s(\varepsilon) \dot{q}_{2}, \dot{q}_{1}+c(\eta) \dot{q}_{2}\right]^{T}$ for the Case III and $\omega_{2}=\left[-s(\eta) s(\varepsilon) \dot{q}_{1}, c(\eta) \dot{q}_{1}+\dot{q}_{2}, s(\eta) c(\varepsilon) \dot{q}_{1}\right]^{T}$ for the Case IV. The corresponding rotational kinetic energy in the global frame can be expressed as $K_{r}=1 / 2 \omega_{2}^{T} I_{2} \omega_{2}$. The inertia matrix in the global frame is $I_{2}=R_{2}{ }^{2} I_{2} R_{2}^{T} \in \mathbb{R}^{3 \times 3}$, where ${ }^{2} I_{2} \in \mathbb{R}^{3 \times 3}$ is the inertia matrix in the body-fixed frame (upper-left submatrix of ${ }^{2} J_{2}$ in Eq. 6). As in Cases I and II, both the total kinetic energy $K=K_{t}+K_{r}$ and the potential energy depend on the GCs $q_{1}, q_{2}$, and the parameters $\eta, \varepsilon$.

The Lagrangian is found as $L=K-V$. By using the Lagrangian in the Lagrange-Euler equations of motion, a system of two coupled second-order differential equations is obtained for the link motion in terms of the GCs $q_{1}$ and $q_{2}$. These equations contain the parameters $\eta$ and $\varepsilon$ and can be written in the form of Eq. (1) by defining the vector of the GCs as $\mathbf{q}=\left[q_{1}, q_{2}\right]^{T}$ and the vector of the GVs as $\dot{\mathbf{q}}=\left[\dot{q}_{1}, \dot{q}_{2}\right]^{T}$. 


\section{SIMULATIONS}

Simulations were performed to investigate the influence of a set of GCs on the dynamic behavior. For selected values of parameters $\eta$ and $\varepsilon$, the dynamic equations of motion were automatically generated using the Lagrangian formalism and the Symbolic Math Toolbox ${ }^{\mathrm{TM}}$ of MATLAB. The obtained nonlinear differential equations were transformed to the form of Eq. (2) suitable for numerical integration. For all four cases, there are singularities at $\eta=0$ and $\eta=\pi$, where the axes of $q_{1}$ and $q_{2}$ coincide. Therefore, by specifying the number of intervals $N \in \mathbb{N}$ and the angular step size $\Delta=\pi / N$, the range of variation for $\eta$ is chosen as $\eta=i \Delta, i=1,2, \ldots, N-1 \in \mathbb{N}$. Similarly, the range for $\varepsilon$ is $\varepsilon=i \Delta, i=-N,-N+1, \ldots, N-1 \in \mathbb{Z}$. The physical parameters were assumed to have the values $m=1, L=1, L_{c}=1, I=0_{3 \times 3}$ corresponding to a simple mass-point spherical pendulum.

The analytical description of a mass-point spherical pendulum oscillation expressed in the spherical coordinate frame was also obtained as

$$
\ddot{q}_{1}=s q_{1} c q_{1} \dot{q}_{2}-\frac{g}{L_{c}} s q_{1}, \quad \ddot{q}_{2}=-2 \frac{s q_{1} c q_{1}}{s q_{1}^{2}} \dot{q}_{1} \dot{q}_{2} .
$$

Here, $q_{1}$ and $q_{2}$ are denoting the polar and azimuthal angles, respectively. The solution of Eq. (9) serves as a baseline case. The root-mean-square (RMS) error $E r$ was computed as $E r=\sqrt{\sum_{i=1}^{N_{\tau}}\left[\left(X_{i}-X_{i, r}\right)^{2}+\left(Y_{i}-Y_{i, r}\right)^{2}+\left(Z_{i}-Z_{i, r}\right)^{2}\right] / N_{\tau}}$ where $X_{i}$ and $X_{i, r}$ are the $x$ components of the center of mass of the pendulum in the global $X Y Z$ frame obtained from a set of GCs and from the baseline model, respectively. The number of time samples is $N_{\tau}$. Each solution of the dynamic equation corresponding to a specific set of GCs was compared to the baseline case through $E r$. Additionally, the simulation time $\tau$ was recorded for each combination of the parameters $\eta$ and $\varepsilon$.

Two different initial conditions (ICs) were considered. The first IC (denoted by IC1) corresponds to the situation where the initial values of GCs are nonzero, i.e., $q_{1} \neq 0, q_{2} \neq 0$, while the initial values of the GVs are zero, i.e., $\dot{q}_{1}=\dot{q}_{2}=0$. The second IC (denoted by IC2), assumes that the initial values of the GCs are zero $q_{1}=q_{2}=0$, while the initial values of the GVs are nonzero. The numerical values of the ICs depend on the selected values of the parameters $\eta$ and $\varepsilon$. Therefore, the correct initial values must be computed for each simulation. For a given initial link position $\mathbf{r}_{2 i}$ and velocity $\mathbf{v}_{2 i}$ in the global reference frame, the corresponding ICs at arbitrary values of $\eta$ and $\varepsilon$ can be found by solving the nonlinear algebraic equations $\mathbf{r}_{2}\left(q_{1}, q_{2}\right)-\mathbf{r}_{2 i}=\mathbf{0}$ and $\mathbf{v}_{2}\left(q_{1}, q_{2}\right)-\mathbf{v}_{2 i}=\mathbf{0}$ for $q_{1}$ and $q_{2}$. The obtained initial values for the GCs are denoted as $q_{1, i}$ and $q_{2, i}$. This task was accomplished using the nonlinear system solver fsolve of MATLAB with the default settings, except that the maximum number of allowed iterations was set to $10^{3}$.

Using the appropriate ICs, the MATLAB function ode45 was utilized to solve nonstiff differential equations and to simulate the system behavior for each combination of $\eta$ and $\varepsilon$. The number of steps used to vary the parameters was chosen to be $N=20$. The duration of the simulation time was set to $20 \mathrm{~s}$, with a sampling interval of $1 \mathrm{~ms}$, i.e., $N_{\tau}=20000$. The relative and absolute tolerance levels were set to $10^{-4}$. The simulations were performed on a ThinkPad notebook with an Intel Core i5-10310U CPU processor and 16 GB RAM.

\section{RESULTS}

From the numerical simulation point of view, the most important factors are the actual simulation time $\tau$ and the accuracy of the simulation results, evaluated by $E r$. The RMS simulation error for the Cases I-IV and two different ICs are shown in Fig. 2. Each sub-figure displays the RMS error $E r$ as a function of the parameters $\eta$ and $\varepsilon$. The gray dots indicate the parameter values for which the ICs were found for a given set of GCs. The colored dots indicate the RMS error value for those parameter values where the dynamic equations were integrated. In regions without colored dots (white background) the ICs allowing the integration were not found. This behavior is expected because the initial link position might not be attainable for some sets of GCs. The set of GCs for which dynamic equations were integrated is a subset of a set of GCs for which ICs 
were found, which in turn is a subset of a set of all possible GC variations. For many sets of GCs with admissible ICs, the corresponding dynamic equations of motion were not integrated due to the divergence of the integration process at a certain simulation instant. The fact the ICs were not found for all combinations of $\eta$ and $\varepsilon$, and that the equations of motion were not integrated for all cases with the correct ICs, shows that a set of GCs influences the system dynamics.

An orthogonal set of GCs does not necessarily result in the smallest $E r$. For instance, for Case I with both IC1 and IC2, Er has smaller values (by a factor of two to four) for the set of nonorthogonal GCs in comparison with the simulations for the set of orthogonal GCs corresponding to $\eta=\pi / 2$ and $\varepsilon= \pm \pi / 2$. This might be explained by different degrees of coupling and interplay of simulation errors in the dynamic equations of motion. For Cases I and III, "clusters" of integrable sets of GCs are grouped around $\eta=\pi / 2$ and $\varepsilon= \pm \pi / 2$ (valid for both ICs). On the other hand, for $\varepsilon=0$ or $\pi$ the dynamic equations were not integrated, which makes sense because for these parameter values the axis of $q_{2}$ coincides with the initial vertical link position and thus becomes a redundant DOF. A similar conclusion can be drawn for the integrable "clusters" for Cases II and IV around $\eta=\pi / 2$ and $\varepsilon=0$ or $\pi$. In these cases, at $\varepsilon= \pm \pi / 2$ dynamic equations were not integrated due to the aforementioned reason. Thus, the obtained results suggest that there is no clear strategy for the selection of the optimal set of GCs that would minimize the RMS error Er. However, by choosing an orthogonal set of GCs we ensure that the obtained model is integrable.

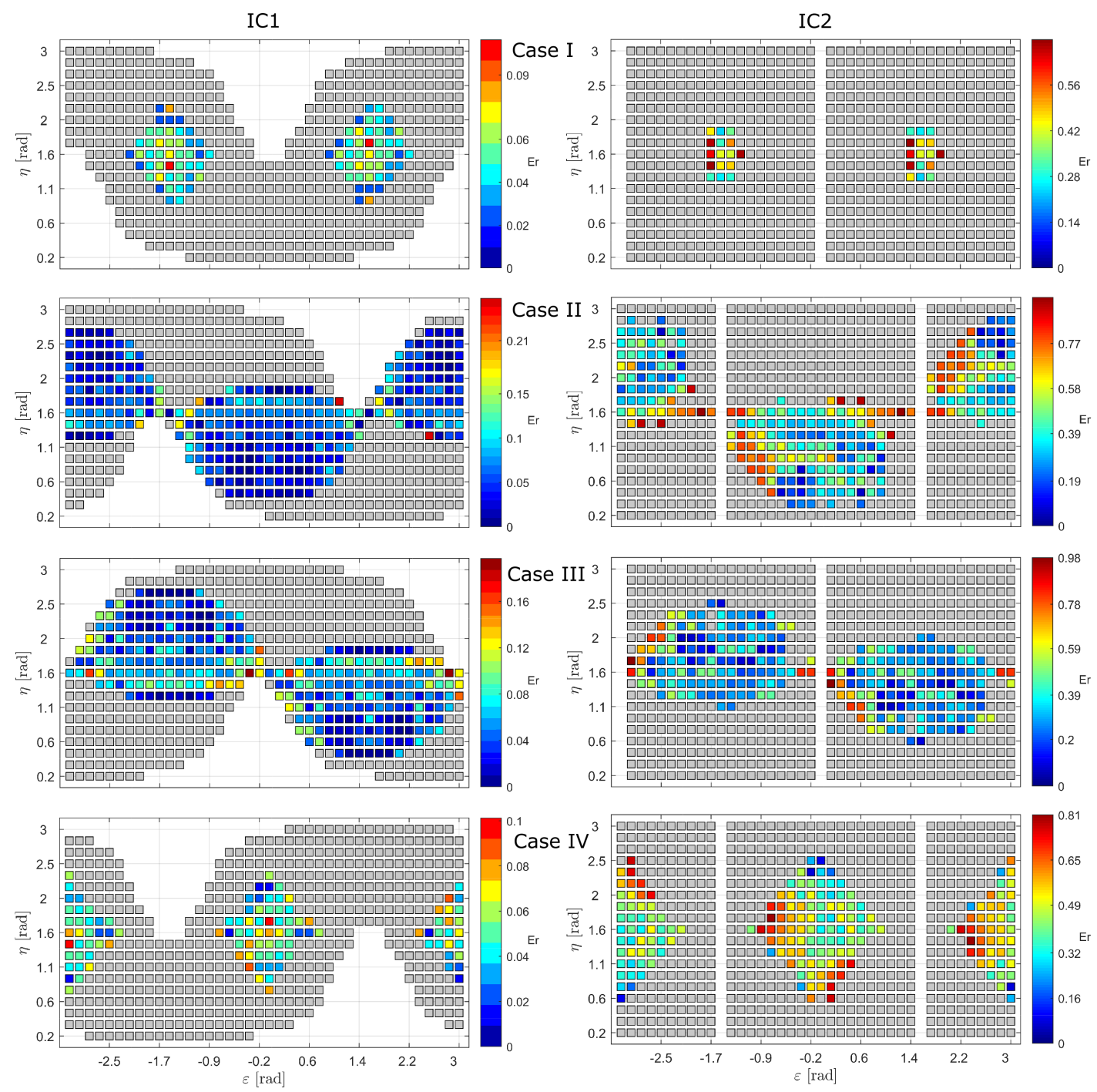

Figure 2: The RMS Er for Cases I-IV with two different ICs. Note that the color bars have different scaling for each subplot. 
IC1
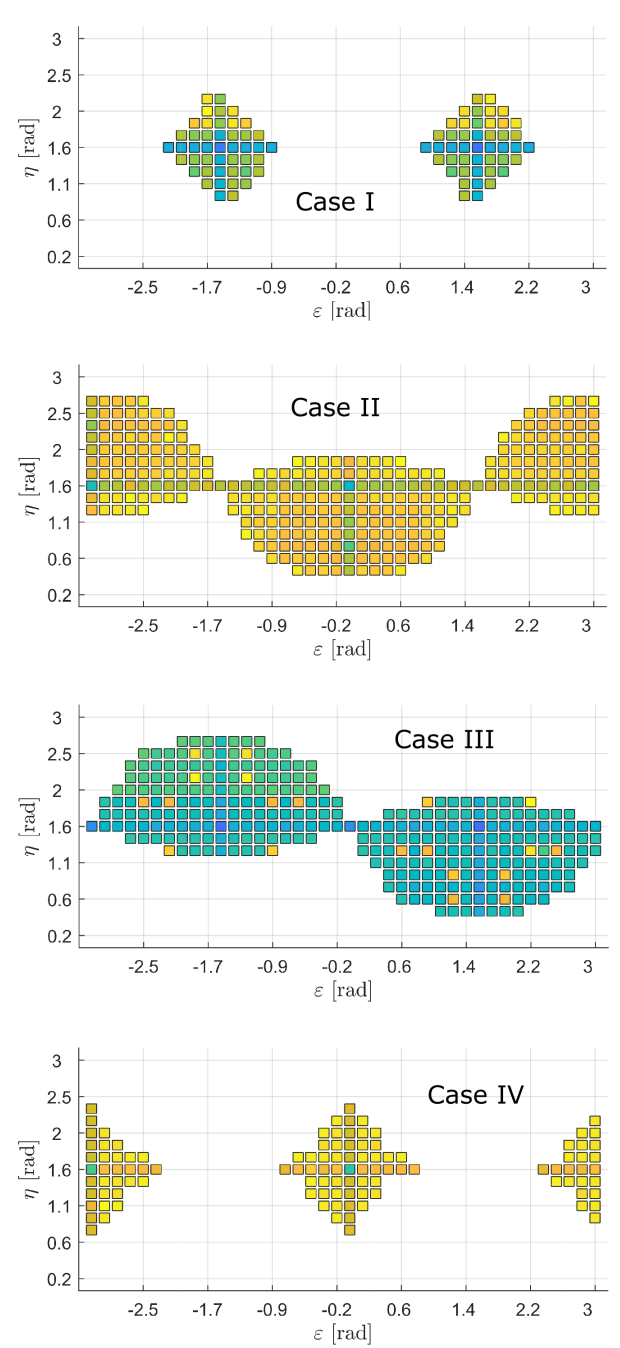

IC2
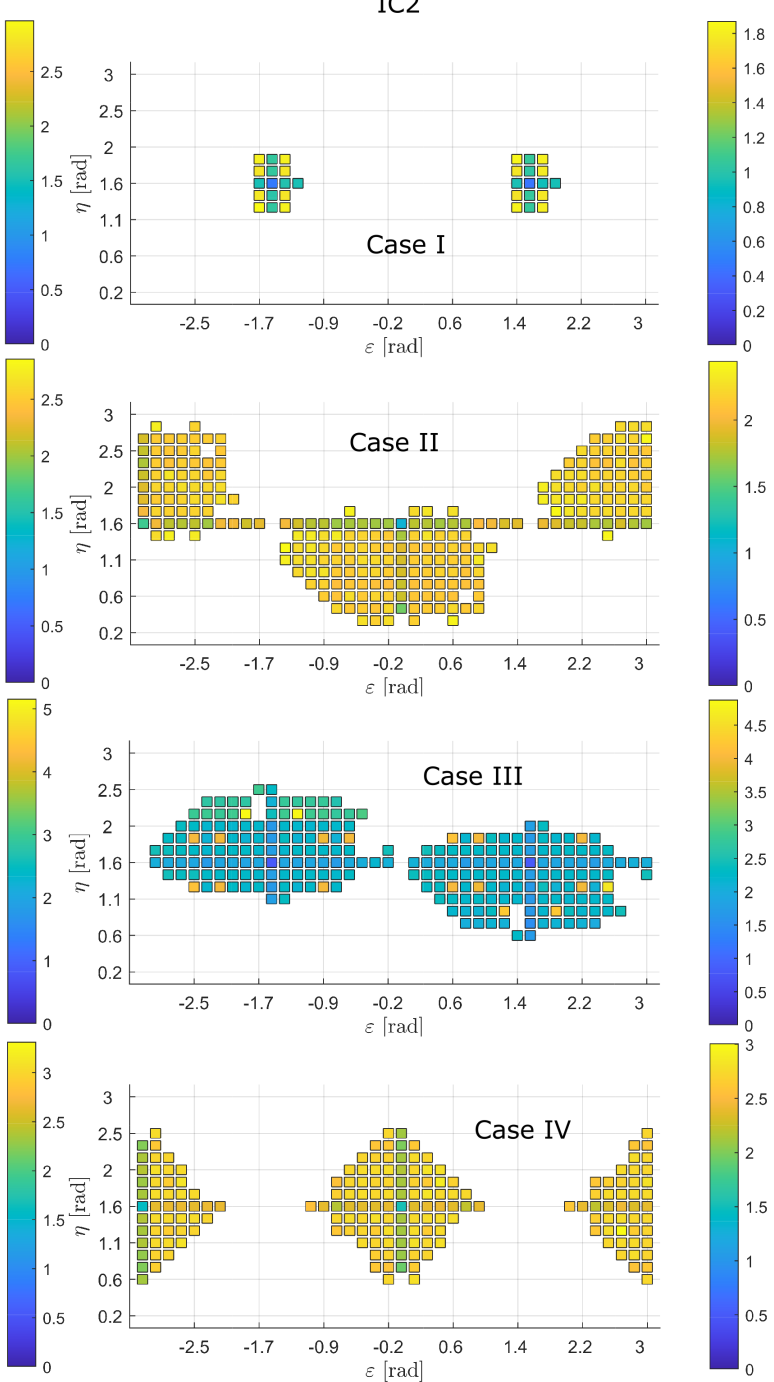

Figure 3: Simulation time $\left(\log _{10}\left(\tau / \tau_{r}\right)\right)$ for Cases I-IV with two different ICs. The color bars have different scaling for each subplot.

Simulation times are shown in Fig. 3 on a logarithmic scale $\log _{10}\left(\tau / \tau_{r}\right)$. Here we can clearly see that the choice of the orthogonal set of GCs leads to a faster simulation. This observation is valid for four cases and for both ICs. For example, simulations with a non-orthogonal set of GCs can require two to five orders of magnitude longer simulation time compared to that with orthogonal counterparts. As a result, the orthogonal sets of GCs $(\eta=\pi / 2$ and $\varepsilon= \pm \pi / 2$ for Cases I and III, $\eta=\pi / 2$ and $\varepsilon=0$ (or $\varepsilon=\pi$ ) for Case II and IV) lead to more efficient simulations. In fact, for the Cases I-IV, the minimum values of the real simulation time are equal to $0.17,0.77,0.46,2.12 \mathrm{~s}$ (IC1) and $0.15,0.63,0.36,1.48 \mathrm{~s}$ (IC2), respectively. Thus, using the local Davenport angles defined for intrinsic rotations leads to faster simulations (by a factor of two to three) compared to the global Davenport angles defined for extrinsic rotations. Finally, it can be observed that using the first GC with an arbitrary rotation axis direction (Cases II and IV) results in slower simulations than using the set of GCs with an arbitrary direction of the second rotation axis (Cases I and III).

In summary, we conclude that a set of GCs affects the description of the system dynamics, simulation error and simulation time. The optimal set of GCs that minimizes the RMS error does not simultaneously minimize the simulation time and vice versa. 


\section{Conclusions}

Different sets of GCs (generalized Euler angles) have been considered from the accuracy point of view and the computation time. It has been shown that there exists a set of orthogonal GCs that allows a fast integration of the equations of motion. However, the use of orthogonal Davenport angles does not necessarily result in the minimum gross simulation error. In certain cases, nonorthogonal Davenport angles yield the minimum gross simulation error. Additionally, it has been observed that the use of intrinsic Davenport angles as GCs leads to more efficient simulations compared to extrinsic Davenport angles.

\section{REFERENCES}

[1] Baruh, H.: Analytical Dynamics. McGraw-Hill (1999)

[2] Goldstein, H.: Classical Mechanics. Addison-Wesley (1980)

[3] Lanczos, C.: The Variational Principles of Mechanics. 4th edn. University of Toronto Press (1970)

[4] Freeman, R.A., Tesar, D.: The generalized coordinate selection for the dynamics of complex planar mechanical systems. Journal of Mechanical Design 104(1) (01 1982) 206-217

[5] Wehage, R.A., Haug, E.J.: Generalized coordinate partitioning for dimension reduction in analysis of constrained dynamic systems. ASME. J. Mech. Des. 104(1) (01 1982) 247-255

[6] Nikravesh, P.E., Haug, E.J.: Generalized coordinate partitioning for analysis of mechanical systems with nonholonomic constraints. ASME. J. Mech., Trans., and Autom. 105(3) (1983) 379-384

[7] Terze, Z., Naudet, J.: Structure of optimized generalized coordinates partitioned vectors for holonomic and non-holonomic systems. Multibody System Dynamics 24(2) (2010) 203-218

[8] Wehage, K.T., Wehage, R.A., Ravani, B.: Generalized coordinate partitioning for complex mechanisms based on kinematic substructuring. Mechanism and Machine Theory 92 (2015) $464-483$

[9] Reungwetwattana, A., Toyama, S.: An efficient dynamic formulation for multibody systems. Multibody System Dynamics 6(3) (2001) 267-289

[10] González, F., Dopico, D., Pastorino, R., Cuadrado, J.: Behaviour of augmented Lagrangian and Hamiltonian methods for multibody dynamics in the proximity of singular configurations. Nonlinear Dynamics 85(3) (2016) 1491-1508

[11] Yang, L., Yao, W., Li, Z.: UK method for solving multi-body system dynamics with singular problems. Advances in Mechanical Engineering 10(4) (2018) 1-13

[12] Impelluso, T.J.: The moving frame method in dynamics: reforming a curriculum and assessment. International Journal of Mechanical Engineering Education 46(2) (2018) 158191

[13] Stephen T. Thornton, J.B.M.: Classical Dynamics of Particles and Systems. 5th edn. Thomson Brooks/Cole (2004)

[14] Davenport, P.B.: Rotations about nonorthogonal axes. AIAA Journal 11(6) (1973) 853-857

[15] Shuster, M.D., Markley, F.L.: Generalization of the Euler angles. J. Astronaut. Sci. 51(2) (2003) 123-132 
[16] Wittenburg, J., Lilov, L.: Decomposition of a finite rotation into three rotations about given axes. Multibody System Dynamics 9(4) (2003) 353-375

[17] Craig, J.J.: Introduction to Robotics, Mechanics and Control. Pearson Prentice Hall (2005)

[18] Abdel-Malek, K.A., Arora, J.S.: Human Motion Simulation, Predictive Dynamics. Elsevier (2013)

[19] Fu, K.S., Gonzalez, R.C., Lee, C.S.G.: Robotics: Control, Sensing, Vision and Intelligence. 5th edn. McGraw-Hill Book Company (1987) 